\title{
An Asymptotic Technique for the Far Field Pattern of a Dipole in an Infinite Stratified Medium
}

\author{
J. T. Rockway, R. J. Marhefka, N. Champagne
}

This article was submitted to

2001 Institute for Electrical and Electronics Engineers Antennas and Propagation Society International Symposium and USNC/URSI National Radio Science Meeting, Boston, MA, July 8-13, 2001

U.S. Department of Energy

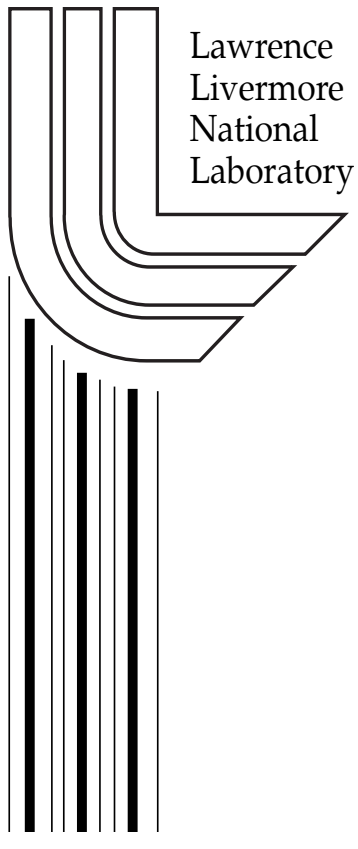

January 8, 2001 


\section{DISCLAIMER}

This document was prepared as an account of work sponsored by an agency of the United States Government. Neither the United States Government nor the University of California nor any of their employees, makes any warranty, express or implied, or assumes any legal liability or responsibility for the accuracy, completeness, or usefulness of any information, apparatus, product, or process disclosed, or represents that its use would not infringe privately owned rights. Reference herein to any specific commercial product, process, or service by trade name, trademark, manufacturer, or otherwise, does not necessarily constitute or imply its endorsement, recommendation, or favoring by the United States Government or the University of California. The views and opinions of authors expressed herein do not necessarily state or reflect those of the United States Government or the University of California, and shall not be used for advertising or product endorsement purposes.

This is a preprint of a paper intended for publication in a journal or proceedings. Since changes may be made before publication, this preprint is made available with the understanding that it will not be cited or reproduced without the permission of the author.

This work was performed under the auspices of the United States Department of Energy by the University of California, Lawrence Livermore National Laboratory under contract No. W-7405-Eng-48.

This report has been reproduced directly from the best available copy.

Available electronically at http://www.doc.gov/bridge

Available for a processing fee to U.S. Department of Energy

And its contractors in paper from

U.S. Department of Energy

Office of Scientific and Technical Information

P.O. Box 62

Oak Ridge, TN 37831-0062

Telephone: (865) 576-8401

Facsimile: (865) 576-5728

E-mail: reports@adonis.osti.gov

Available for the sale to the public from

U.S. Department of Commerce

National Technical Information Service

5285 Port Royal Road

Springfield, VA 22161

Telephone: (800) 553-6847

Facsimile: (703) 605-6900

E-mail: orders@ntis.fedworld.gov

Online ordering: http://www.ntis.gov/ordering.htm

OR

Lawrence Livermore National Laboratory

Technical Information Department's Digital Library

http://www.llnl.gov/tid/Library.html 


\title{
An Asymptotic Technique for the Far Field Pattern of a Dipole in an Infinite Stratified Medium
}

\author{
Jeanne T. Rockway ${ }^{1}$, Ronald J. Marhefka ${ }^{1}$, and Nathan J. Champagne ${ }^{2}$ \\ The Ohio State University ${ }^{1} \quad$ University of California $^{2}$ \\ ElectroScience Laboratory Lawrence Livermore National Laboratory \\ 1320 Kinnear Road \\ Columbus, OH 43212-1191 \\ 7000 East Ave., L-154 \\ Livermore, CA 94550-9516
}

\section{Introduction}

Modern antennas especially arrays are being placed in layers of materials on complex environments. This technique produces aesthetically pleasing structures if necessary, allows for more freedom in structure planning, and can improve antenna performance. In the past, buried antennas have been studied by numerous authors such as in Reference [1]. Recent work on this subject uses spectral and/or numerical moment method formulations [2, 3, 4]. For high frequency analysis it is important to find efficient and accurate methods for design purposes.

A rigorous recursive method for plane waves reflection and transmission coefficients by Richmond [5] has been used in the past for dipoles above multilayer slabs [6]. This solution is modified in this paper to account for forward and backward traveling rays with appropriate spread factors for a dipole in the media. Extensive validation for this approximate method shows good agreement with a Method of Moments code. This code is developed at Lawrence Livermore National Laboratory [4]. The geometry for these comparisons uses a dipole in nontruncated dielectric multilayer slabs.

\section{Theoretical Approach}

One approach to the analysis of large antennas in complex environments is to approximately separate the detailed antenna design phase from the siting calculations. This so-called first order hybrid approach has been used for many years for perfectly conducting structures. For example, Method of Moments (MoM) can be used to analyze an antenna in free space or over a ground plane. The currents or tabulated antenna pattern is transferred to a Uniform Geometrical Theory of Diffraction (UTD) code. It analyzes the effects of the structure. Recently, there is an increased need to place the antennas near or in dielectric

\footnotetext{
${ }^{\dagger}$ Work performed at the Lawrence Livermore National Laboratory under the auspices of the U.S. Department of Energy under Contract W-7405-ENG-48.
} 
materials. This arises from a desire to hide a wireless communication antenna in a building [7] or place arrays on ships or aircraft.

An efficient analysis of the case of an antenna outside multilayer material slabs joined at arbitrary angles has been developed for this purpose [6] and implemented into a UTD code [8]. The reflection and transmission effects have been calculated using a rigorous plane wave solution by Richmond [5]. In this method, the forward and backward traveling waves within the material are found by a recursion technique. These coefficients are then applied to near zone applications by using UTD techniques. This approximation works well where surface waves are not important, i.e., when the antennas are not too near the surface and the observation point is not near grazing to the material. In practice, however, the region of applicability for engineering applications has been shown to be quite extensive.

This paper presents the extension of this simple and efficient method for an antenna inside multilayer materials. The fields are decomposed into two parts for calculation purposes. There is a "direct ray" from the antenna to the observer that contains the effects of the composite field traveling "forward" through the medium. It is determined by using the recursive method to find the "transmitted" field from the observer layer and then stopping at the antenna layer. There is also a ray that initially travels in the "backward" direction, is then reflected from the bottom of the material and then proceeds in the "forward" direction. It is determined by using the "reflected" field and combining it with the above mentioned "transmitted" field. The coefficients found from the recursive method need to be augmented by spread factors at the boundaries. In the case of the dipole and observer outside the material, these spread factors balance out to unity. This is not necessarily the case when the antenna is in one of the layers.

In the early design phases or in the siting phase when the antenna is placed in a complex environment, the pattern factors or the MoM calculated currents can be used in this solution for fast results. In general, this approach works well where the dominant fields are optical in nature. Surface waves need to be added for finite slabs in regions where the diffractions from the ends are relatively strong. However, in order to test the fundamental optics solution for the infinite slab, comparisons are made with a MoM solution using a nontruncated slab Green's function as discussed in the section below.

\section{Numerical Results}

The asymptotic technique is compared for multiple cases to a method of moments solution, the EIGER code [4], by Lawrence Livermore National Laboratory (LLNL). In the following figures, the frequency is 300 megahertz. Figure 1 shows the far field pattern for a half-wave horizontal dipole in the middle layer of three different materials. The layer, L1, has a relative permittivity of 5 with a width of 3 meters. The layer, L3, has a relative permittivity of 2 with width of 3 
meters. The dipole layer, L2, has a relative permittivity of 3 with width of 5 meters. The dipole is 2 meters right of the interface between L1 and L2. The geometry for Figure 2 consists of a half-wavelength (free-space wavelengths) horizontal dipole in a dielectric half-space medium of 5 . The antenna is 3 meters to the left of L2, a 6 meter wide dielectric layer of permittivity of 7. Good agreement between the two techniques may be seen from the plots.

\section{Conclusions}

An efficient method to find the far fields of a dipole in a stratified medium has been studied. It is based on a recursive technique for plane wave reflection and transmission coefficients modified with a spherical spread factor. The present development produces a quick and accurate solution where surface waves are not important. It has been extensively validated with a Method of Moments solution for a non-truncated slab, thereby eliminating surface wave considerations. This ray based solution works well for antenna positions not on the material boundary or for very thin slabs.

\section{References}

[1] A. W. Briggs, "Radiation Fields from a Horizontal Electrical Dipole in a Semi-Infinite Conducting Medium," IRE Trans. Ant. Prop., pp. 358-362, July 1962.

[2] E. K. Miller and F. J. Deadrick, "Analysis of Wire Antennas in the Presence of a Conducting Half-Space: Part III - The Buried Antenna," Technical Report UCRL-52228, Lawrence Livermore National Laboratory, February 1977.

[3] J. J. van Tonder, J. H. Cloete, "The Numerical Analysis Of Planar Antennas Buried In Layered Media," The Transactions of the South African Institute of Electrical Engineers, Vol. 88, No. 3, pp. 82-88, September 1997.

[4] R. M. Sharpe, J. B. Grant, N. J. Champagne, W. A. Johnson, R. E. Jorgenson, D. R. Wilton, W. J. Brown, J. W. Rockway, "EIGER: Electromagnetic Interactions GenERalized," IEEE AP-S/URSI Int. Symp., Montreal, July 1997.

[5] J. H. Richmond, "Efficient Recursive Solutions For Plane And Cylindrical Multilayers," Technical Report 1968-1, The Ohio State University ElectroScience Laboratory, Dept. of Elec. Engr., August 1965.

[6] A. Aktas and R. J. Marhefka, "An Approximate UTD Coefficient for Material Plate Junctions," IEEE AP-S/URSI Int. Symp., Atlanta, June 1998.

[7] R. J. Marhefka, "High Frequency Spatial Coupling in a Complex Environment," IEEE AP-S/URSI Int. Symp., Baltimore, July 1996.

[8] R. J. Marhefka, "Numerical Electromagnetics Code - Basic Scattering Code (NEC-BSC Version 4.2), Preliminary User's Manual," Technical Report, The Ohio State University ElectroScience Laboratory, October 2000. 


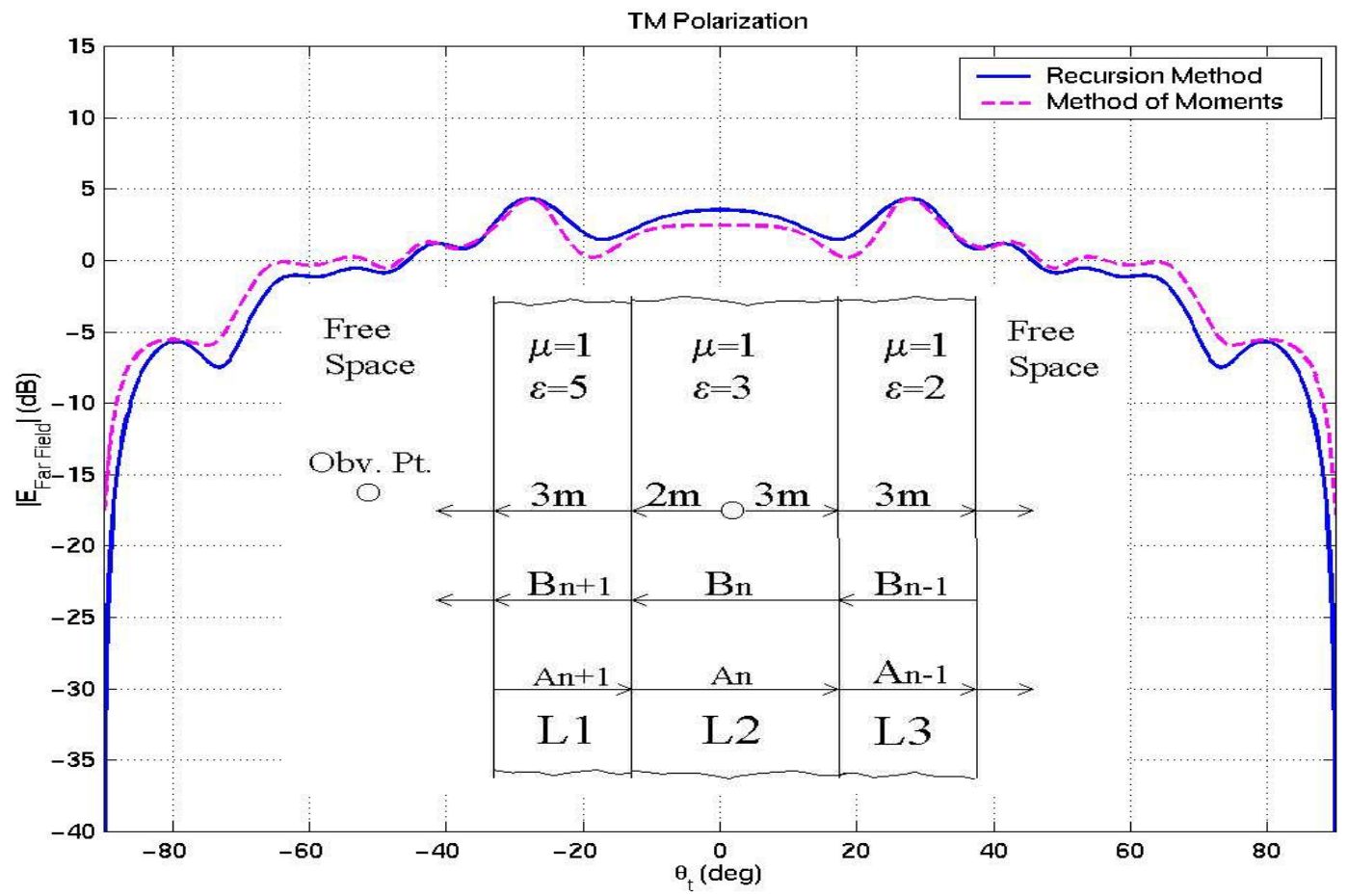

Figure 1: Far field pattern for a dipole embedded in the center layer of a three laver infinite slab.

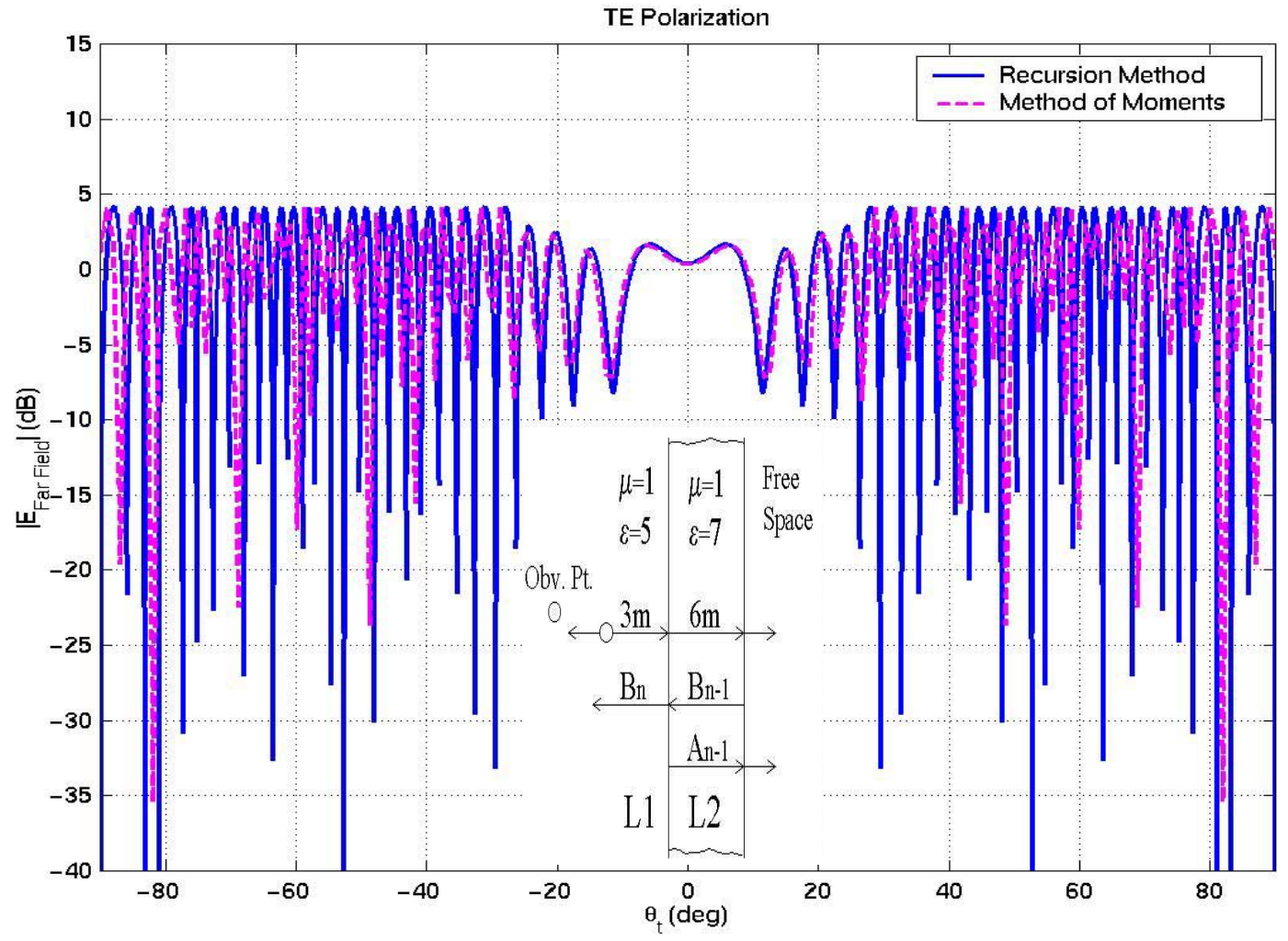

Figure 2: Far field pattern for a dipole in a dielectric half-space next to a thick infinite layer. 